\title{
PÓS-TRATAMENTO FÍSICO-QUÍMICO POR FLOTAÇÃO FAD EM EFLUENTES DE LATICÍNIOS
}

\author{
Gabriel Taccolini Papp ${ }^{1 *}$, Andressa Corso ${ }^{2}$, Juliana Bortoli Rodrigues Mees ${ }^{3}$ \\ ${ }^{1}$ Programa de Pós-Graduação Engenharia de Segurança do Trabalho, Universidade de Mogi das Cruzes, Mogi das Cruzes, Brasil. \\ ${ }^{2}$ Programa de Pós-Graduação em Tecnologias Ambientais, Universidade Tecnológica Federal do Paraná, Medianeira, Brasil. \\ ${ }^{3}$ Departamento Acadêmico de Engenharia Ambiental, Universidade Tecnológica Federal do Paraná, Medianeira, Brasil.
}

*E-mail: gabrieltpapp@gmail.com

Recebido em:26/06/2018

Aceito em:30/07/2019

\section{RESUMO}

A agroindústria de laticínios constitui um setor com uma geração de efluente de alto potencial poluidor, que sem o tratamento adequado acaba se tornando um problema ambiental. Buscando solucionar os efeitos do tratamento inadequado destes efluentes, complementar ao tratamento convencional, em alguns casos é realizada a aplicação de um pós-tratamento visando uma melhor qualidade do efluente final. Uma das formas utilizadas é a flotação precedida de coagulação/floculação, que através de agentes coagulantes e floculantes permitem à aglomeração de partículas possibilitando a flotação. Essas substâncias utilizadas são produtos químicos normalmente à base de alumínio ou ferro, acarretando na geração de lodos com altas concentrações de íons metálicos, dificultando e encarecendo a destinação do lodo. Tendo em vista os problemas citados, vêm sendo estudados e utilizados coagulantes e floculantes com base orgânica, uma vez que estes geram lodos biodegradáveis. Assim, este trabalho teve por objetivo comparar a eficiência entre dois coagulantes, sendo um inorgânico (Klaraid IC 1176L) e o outro orgânico (Novfloc 1540), associados ao processo de flotação e sedimentação em escala de bancada, no tratamento de efluente de uma agroindústria de laticínio. Para a realização deste trabalho, foi aplicada a metodologia de planejamento experimental e otimização de processos, utilizando como variáveis independentes a concentração de coagulantes e floculantes, tendo como variável resposta à remoção de turbidez. Como primeiro passo foi feito o planejamento inicial fatorial $2^{2}$ de sedimentação e flotação visando ajustar as faixas de aplicação, seguindo para um delineamento composto central rotacional visando à otimização dos processos da sedimentação e flotação. Com a utilização do coagulante de base inorgânica (Klaraid) seguido pelo floculante, obteve-se uma remoção de turbidez de até 78,93\% para o processo de sedimentação, já para o processo de flotação os melhores resultados apontaram uma remoção de turbidez de 74,92\%. Com o uso do coagulante de base orgânica (Novfloc) combinado ao uso do floculante, a eficiência de remoção de turbidez para sedimentação chegou até 78,09\%, já na flotação o resultado foi até $72,11 \%$ de remoção.

Palavras-chave: Coagulação. Floculação. Flotação. Efluente. Laticínios.

\section{Introdução}

$\mathrm{O}$ alto crescimento populacional da sociedade requer uma maior demanda de água, que após a utilização sofre alterações em suas características físico-químicas, acarretando na geração de efluentes. A indústria de laticínios, comparada com algumas outras indústrias, tem o consumo de água elevado, consequentemente uma alta geração de efluentes. Segundo Villa, Silva e Nogueira [1] os efluentes líquidos gerados em uma agroindústria de laticínios apresentam elevados valores de DBO e DQO devido às altas concentrações de carboidratos, proteínas e lipídios. Santos et al [2] ressaltam que os constituintes desse efluente incluem em geral: detergentes, desinfetantes, açúcares, que passam a ser diluídos nas lavagens dos equipamentos e demais instalações da indústria.
Para o tratamento de águas residuárias provenientes de agroindústrias, geralmente se faz o uso de processos biológicos, combinados a processos físicos e físico-químicos [3]. Para o tratamento físico-químico é comum o auxílio de coagulantes inorgânicos a base de ferro ou alumínio, devido ao seu custobenefício e sua eficiência [4]. Em contrapartida, avaliando este uso do ponto de vista ambiental, esses tipos de coagulantes trazem consigo alguns pontos negativos, pois além de levar resquícios de sais de ferro e alumínio no lodo, esses coagulantes podem ser tóxicos e não são biodegradáveis [5]. Desta maneira os lodos gerados a partir destes tipos de coagulantes se toram um problema e necessitam de tratamento e uma disposição final correta.

Visando minimizar estes efeitos em estações de tratamento de águas, líquidos industriais e esgotos, tem-se como opção a substituição dos coagulantes inorgânicos por 
coagulantes orgânicos sendo estes biodegradáveis, menos agressivos e não tóxicos. Assim o lodo gerado, devido às suas características, pode ser destinado ao uso agrícola como a adubação e recuperação de áreas degradadas, sem apresentar riscos de contaminação [6].

\section{Metodologia}

O presente estudo foi desenvolvido utilizando efluente líquido industrial proveniente de uma agroindústria de laticínios, com as seguintes características: elevadas concentrações de DBO e DQO, devido as elevadas quantidades de proteínas, carboidratos e lipídeos em sua composição.

O experimento foi desenvolvido em escala de bancada, onde avaliou-se o emprego de coagulantes orgânicos e inorgânicos, comparando os processos de coagulação/floculação seguidos de sedimentação e de flotação por ar dissolvido (FAD). O coagulante inorgânico utilizado foi o Klaraid IC 1176L, mais conhecido como Policloreto de Alumínio, o qual é um coagulante catiônico que possui baixo peso molecular. Já o coagulante com base orgânica utilizado no trabalho foi o Novfloc 1540, o qual é um polieletrólito catiônico derivado das aminas, resinas poliquaternárias e sais orgânicos. O floculante usado foi o mesmo para ambos os coagulantes, Polfloc D150 o qual é um polieletrólito de longa cadeia molecular, medianamente catiônico, derivado da poliacrilamida.

Foram necessários ensaios preliminares, para a determinação da dosagem mínima de coagulante e a determinação do pH ótimo de coagulação. $\mathrm{O}$ teste de dosagem mínima de coagulante teve como princípio básico, identificar a menor dosagem de cada coagulante necessária para a formação de pequenos flocos. Os valores de concentrações utilizadas nos ensaios de dosagem mínima seguem abaixo na Tabela 1. Analisaram-se seis concentrações em béqueres de $600 \mathrm{~mL}$, com $500 \mathrm{~mL}$ de efluente, onde os béqueres foram submetidos à agitação lenta de $30 \mathrm{rpm}$, no equipamento Jar Test até a visualização de pequenos flocos, caracterizando esta visualização como dosagem mínima.

Tabela 1 -Concentrações utilizadas para o teste de dosagem mínima.

\begin{tabular}{c|cccccc}
\hline Coagulantes & \multicolumn{5}{c}{ Concentrações utilizadas $\left(\mathbf{p p m} / \mathbf{m g ~ L}^{-1}\right)$} \\
\hline Ensaios & 1 & 2 & 3 & 4 & 5 & 6 \\
\hline Novfloc & 20 & 40 & 60 & 80 & 100 & 120 \\
Polfloc & 40 & 60 & 80 & 100 & 120 & 140 \\
\hline
\end{tabular}

Em seguida, foi utilizada a concentração mínima para a determinação do $\mathrm{pH}$ ótimo de coagulação, testaram-se as seguintes faixas de $\mathrm{pH}: 6,0 ; 6,5 ; 7,0 ; 7,5 ; 8,0 ; 8,5$. Todos os valores estão dentro da faixa recomendada para o uso destes coagulantes. Para a correção do $\mathrm{pH}$, foi feita a adição de $\mathrm{HCl} 1 \mathrm{e}$
0,1 mol. $\mathrm{L}^{-1}$, e NaOH 1 e $0,1 \mathrm{~mol} . \mathrm{L}^{-1}$. Os ensaios ocorreram em béqueres de $600 \mathrm{~mL}$, com $500 \mathrm{~mL}$ de efluente, onde os testes foram submetidos à agitação rápida de 100 rpm por 20 segundos, agitação lenta de $30 \mathrm{rpm}$ por 15 minutos e sedimentação por 30 minutos. A partir desses testes avaliou-se a faixa de $\mathrm{pH}$ que obteve a melhor eficiência de coagulação com relação à redução de cor e turbidez.

Para a realização dos ensaios experimentais, foi utilizada a metodologia de planejamento experimental e a otimização de processos. Como planejamento inicial foram realizados 4 planejamentos fatoriais $2^{2}$ (dois para sedimentação e dois para flotação), com 4 pontos fatoriais, 3 pontos centrais. As variáveis independentes, nos dois planejamentos foram as concentrações de coagulantes e floculantes, tendo como variável resposta à remoção da turbidez.

Os ensaios de coagulação, floculação e sedimentação foram realizados no equipamento Jar Test, o qual possui agitadores que permitem a simulação da mistura rápida e da floculação. Os ensaios ocorreram nos jarros de 2 litros, contendo 1,5 litros de efluente em cada jarro. As condições adotadas para os ensaios foram adotadas com base na bibliografia: para coagulação $100 \mathrm{rpm}$ por 20 segundos, para floculação $30 \mathrm{rpm}$ por 15 minutos Di Bernardo, Di Bernardo e Centurione [7].

Com o planejamento inicial foi possível avaliar os efeitos da variável resposta e a necessidade de se ampliar a faixa de estudo. Como passo seguinte foi realizado o delineamento composto central rotacional (DCCR), que reduz o número de repetições e melhora a qualidade da informação, gerando equações de segundo grau e gráficos de superfície, dos quais pode-se avaliar e definir uma faixa aproximada ótima do uso de coagulantes e floculantes, para se obter uma melhor remoção de turbidez. O DCCR contou com 4 pontos fatoriais, 3 pontos centrais e 4 pontos axiais. Na Tabela 2 mostra-se os níveis codificados e reais das variáveis independentes do delineamento composto para sedimentação.

Tabela 2 - Níveis reais e codificados do DCCR para sedimentação.

\begin{tabular}{c|ccccc}
\hline $\begin{array}{c}\text { Variáveis Independentes } \\
(\mathbf{p p m})\end{array}$ & \multicolumn{5}{|c}{$\begin{array}{c}\text { Níveis reais e codificados das variáveis } \\
\text { independentes }\end{array}$} \\
\hline & $\mathbf{- 1 , 4 1}$ & $\mathbf{- 1}$ & $\mathbf{0}$ & $\mathbf{1}$ & $\mathbf{1 , 4 1}$ \\
\cline { 2 - 7 } Klaraid & 173 & 210 & 300 & 390 & 427 \\
Polfloc & 4,8 & 6 & 9 & 12 & 13,3 \\
\hline & $\mathbf{- 1 , 4 1}$ & $\mathbf{- 1}$ & $\mathbf{0}$ & $\mathbf{1}$ & $\mathbf{1 , 4 1}$ \\
\cline { 2 - 7 } Novfloc & 173 & 210 & 300 & 390 & 427 \\
Polfloc & 2,8 & 4 & 7 & 10 & 11,3 \\
\hline
\end{tabular}

Os ensaios de coagulação, floculação e flotação foram realizados com o auxílio do equipamento Flota Test, este equipamento possui uma câmara de pressão e jarros que permitem a introdução de ar saturado para flotação, um equipamento Jar Test que auxilia na coagulação e floculação e um compressor de ar. Os ensaios ocorreram em jarros de 2 litros 
com 1,5 litros de efluente. Para a flotação, foi realizada a introdução de efluente com ar saturado com um volume de $20 \%$ da cuba.

Para todos os ensaios de flotação, os seguintes parâmetros foram fixados: gradiente de mistura rápida igual a $100 \mathrm{rpm}$; tempo de mistura rápida igual a $20 \mathrm{~s}$; gradiente de floculação igual a $30 \mathrm{rpm}$; tempo de floculação igual a 15 min baseado no trabalho de Solana [8]. Já os parâmetros de pressão de saturação de 4 bar; taxa de recirculação igual a 20\%; Tc igual de 60 s; velocidade de flotação igual a $10 \mathrm{~cm} \cdot \mathrm{min}^{-1}$ foram baseados no trabalho de Centurione Filho e Di Bernardo [9]. O tempo de coleta (Tc) foi calculado dividindo a altura de saída do jarro pela velocidade de flotação.

Como passo seguinte também foi feito o delineamento composto central rotacional (DCCR) para a flotação que reduz o número de repetições e melhora a qualidade da informação (Tabela 3).

Tabela 3 - Níveis reais e codificados do DCCR para flotação.

\begin{tabular}{c|ccccc}
\hline $\begin{array}{c}\text { Variáveis Independentes } \\
\text { (ppm) }\end{array}$ & \multicolumn{5}{|c}{$\begin{array}{c}\text { Níveis reais e codificados das variáveis } \\
\text { independentes }\end{array}$} \\
\hline & $\mathbf{- 1 , 4 1}$ & $\mathbf{- 1}$ & $\mathbf{0}$ & $\mathbf{1}$ & $\mathbf{1 , 4 1}$ \\
\cline { 2 - 7 } Klaraid / Novfloc/ & 173 & 210 & 300 & 390 & 427 \\
Polfloc & 5,8 & 7 & 10 & 13 & 14,3 \\
\hline
\end{tabular}

O software STATISTICA, versão 10.0 (Statsoft ${ }^{\mathrm{TM}}$, USA), foi utilizado para a determinação dos efeitos das variáveis independentes, análise de variância (ANOVA), o cálculo dos coeficientes da regressão e para obtenção dos gráficos de superfícies de resposta e de contorno, todos ao nível de $5 \%$ de significância.

\section{Resultados e discussões}

\subsection{Resultados dos ensaios preliminares}

Os testes de dosagem mínima de coagulante, apresentaram resultados de 100 ppm para ambos os coagulantes, considerando o ponto de dosagem mínima à visualização dos primeiros flocos formados.

Para o coagulante Klaraid $1176 \mathrm{~L}$ a dosagem de 100 ppm, também foi mais alta que a apresentada por Fontanella [10], que necessitou de 50 ppm para visualizar a dosagem mínima. O que pode ser explicado também pela diferença de efluente trabalhado, que no caso do trabalho referenciado foi oriundo de indústria de cerâmica, com valores de turbidez muito menores.

Para o teste de determinação de $\mathrm{pH}$ ótimo, os ensaios que apresentaram melhor remoção de turbidez, para ambos os coagulantes foram os ensaios com pH igual a 7,5, apresentando remoções de $65,1 \%$ para o coagulante Novfloc e $44,67 \%$ para o coagulante Klaraid. Assim para os planejamentos experimentais realizou-se o ajuste de $\mathrm{pH}$ do efluente para a faixa que se teve maior eficiência na remoção de turbidez, isto é, com pH 7,5.

\subsection{Delineamento Composto Central Rotacional de Sedimentação}

Visando à otimização das condições experimentais, com base no planejamento inicial, utilizou-se um DCCR. Para o início da execução do delineamento composto, a faixa de estudo foi deslocada e em relação ao fator floculante a faixa foi deslocada apenas para o Klaraid com base nos resultados dos ensaios preliminares e em busca de uma representatividade maior dos resultados encontrados de remoção de turbidez. A matriz experimental para o DCCR é ilustrada na Tabela 4, com os níveis e a variável resposta, nos 11 ensaios.

Tabela 4 - Matriz do DCCR para sedimentação (níveis reais e codificados).

\begin{tabular}{|c|c|c|c|c|c|c|c|}
\hline & Ensaios & Novfloc (ppm) & Polfloc (ppm) & Rem. Turb (\%) & Klaraid (ppm) & Polfloc (ppm) & Rem. Turb (\%) \\
\hline \multirow{4}{*}{ Pontos fatoriais } & 1 & $210(-1)$ & $4(-1)$ & 63,44 & $210(-1)$ & $6(-1)$ & 63,12 \\
\hline & 2 & $390(1)$ & $4(-1)$ & 75,95 & $390(1)$ & $6(-1)$ & 74,37 \\
\hline & 3 & $210(-1)$ & $10(1)$ & 70,74 & $210(-1)$ & $12(1)$ & 67,95 \\
\hline & 4 & $390(1)$ & $10(1)$ & 77,58 & $390(1)$ & $12(1)$ & 76,56 \\
\hline \multirow{3}{*}{$\begin{array}{l}\text { Repetição no } \\
\text { ponto central }\end{array}$} & 5 & $300(0)$ & $7(0)$ & 73,07 & $300(0)$ & $9(0)$ & 71,91 \\
\hline & 6 & $300(0)$ & $7(0)$ & 73,21 & $300(0)$ & $9(0)$ & 72,42 \\
\hline & 7 & $300(0)$ & $7(0)$ & 72,6 & $300(0)$ & $9(0)$ & 72,19 \\
\hline \multirow{4}{*}{ Pontos axiais } & 8 & $173(-1,41)$ & $7(0)$ & 64,51 & $173(-1,41)$ & $9(0)$ & 66,33 \\
\hline & 9 & $427(1,41)$ & $7(0)$ & 78,09 & $427(1,41)$ & $9(0)$ & 78,93 \\
\hline & 10 & $300(0)$ & $2,8(-1,41)$ & 70,14 & $300(0)$ & $4,8(-1,41)$ & 71,53 \\
\hline & 11 & $300(0)$ & $11,3(1,41)$ & 73,63 & $300(0)$ & $13,3(1,41)$ & 75,81 \\
\hline
\end{tabular}

A remoção da turbidez foi maior que no planejamento inicial, chegando a remoções de $78,09 \%$ e $78,93 \%$ com o
Novfloc e Klaraid respectivamente. Com base nos resultados obtidos na Tabela 3, ajustaram-se os dados para obtenção de um 


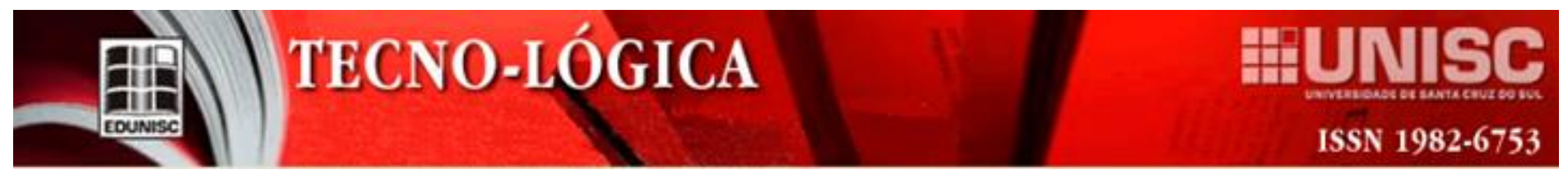

modelo quadrático, relacionando a eficiência de remoção de turbidez. Os modelos ilustrados nas Eq.1 e Eq.2 apresentaram $\mathrm{R}^{2}=0,93248$ e $\mathrm{R}^{2}=0,98929$, significando que $93,25 \%$ e $98,92 \%$ da variação na eficiência de remoção de turbidez é explicada pelo modelo.

Rem. Turbidez pelo Klaraid $=72,17+4,71$ Coag $+1,64$ Floc - 0,66 Coag x Floc (Eq. 1)

Rem. Turbidez pelo Novfloc $=72,97+4,82$ Coag $+1,74$ Floc - 1,42 Coag x Floc - 0,747 Coag ${ }^{2}$ (Eq. 2)

De acordo com a Figura 1, pode-se visualizar o gráfico da probabilidade normal dos resíduos para ambos os casos, que apresentam ajuste satisfatório, pois nos dois casos todos os

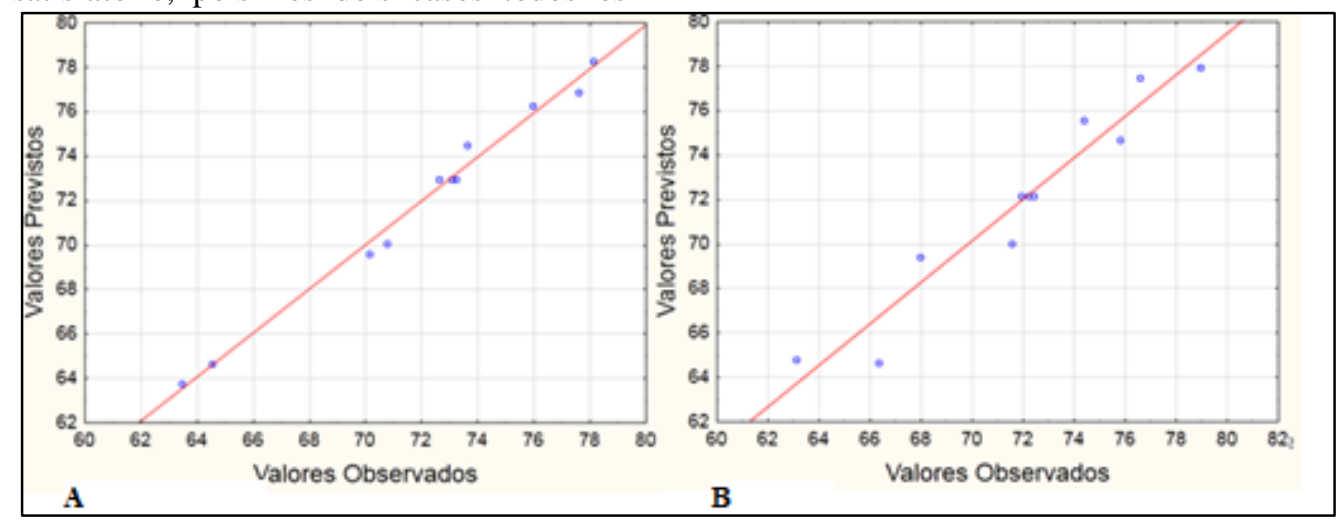

Figura 1 - Gráfico de Probabilidade Normal dos Resíduos (A - Novfloc) (B - Klaraid).
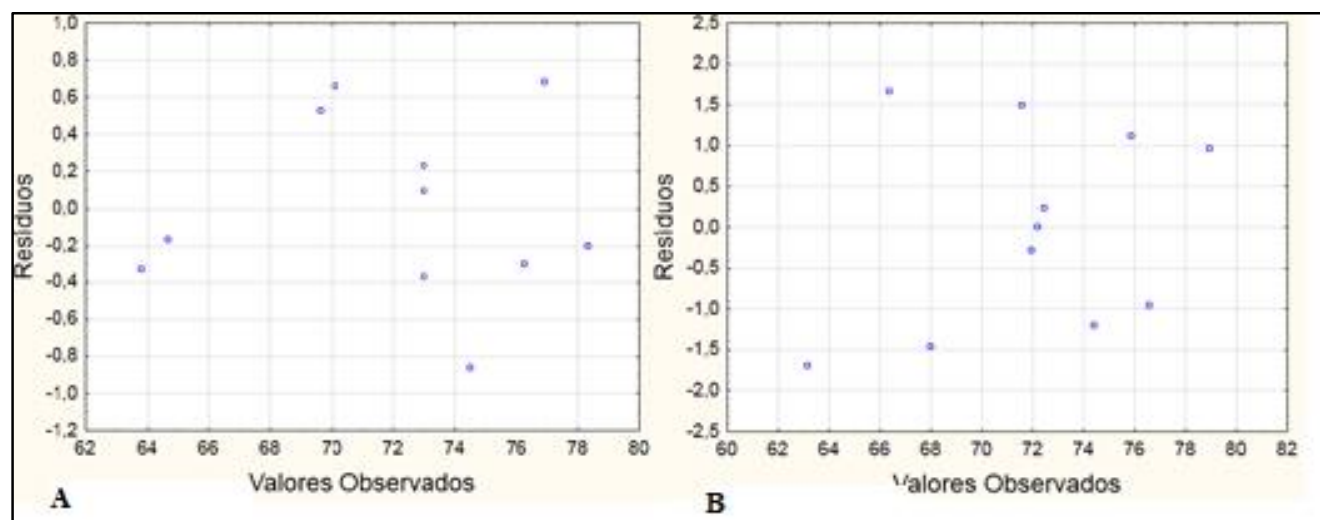

Figura 2 - Gráfico dos Resíduos pelos Valores Observados (A - Novfloc) (B - Klaraid). resíduos estão distribuídos ao redor da reta. Já na Figura 2, apresenta-se o gráfico dos resíduos pelos valores observados, onde, pode-se visualizar que os valores se apresentam de forma aleatória, sem qualquer tendência.

As estimativas, dos efeitos relacionados à eficiência de remoção de turbidez são ilustradas nos diagramas de Pareto (Figura 3). Os efeitos além da linha tracejada são considerados significativos em um intervalo de $95 \%$ de confiança. 

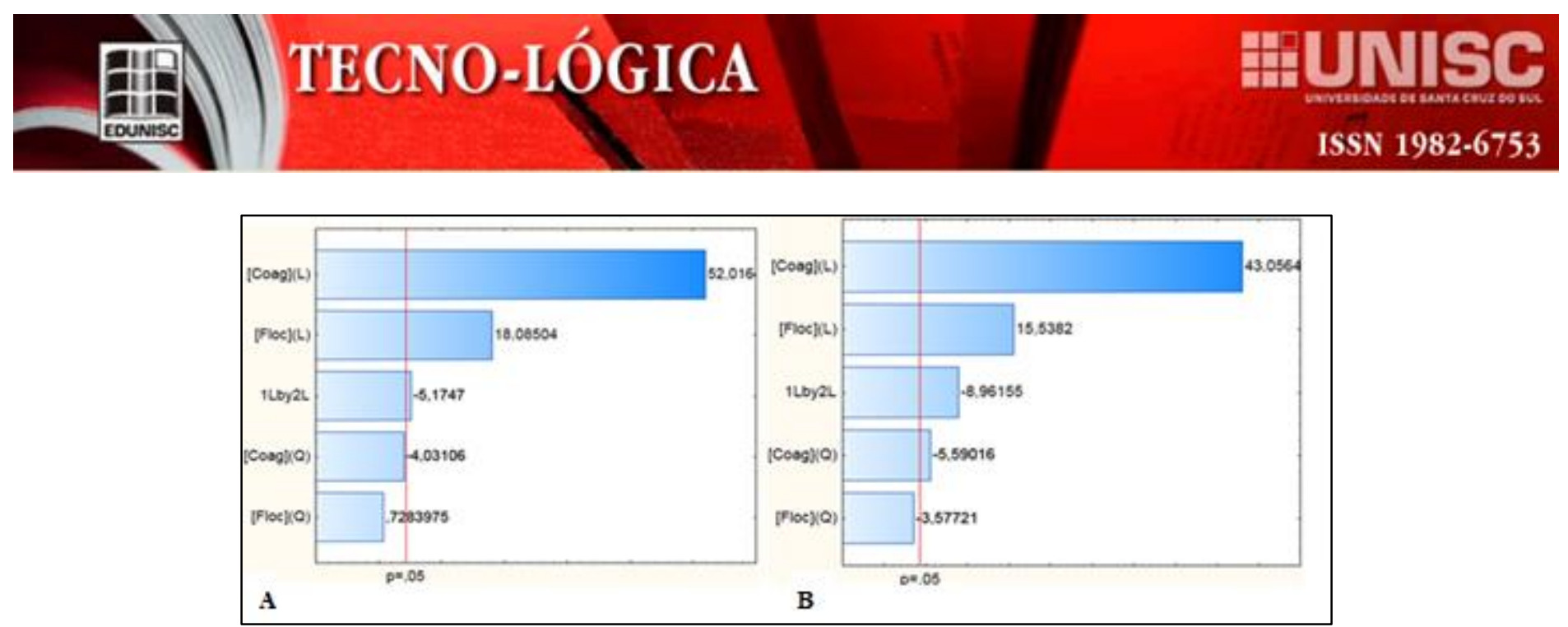

Figura 3 - Diagrama de Pareto (A - Para o Klaraid) (B - Para o Novfloc).

Com base na Figura 3-A e Figura 3-B, pode-se visualizar que, para o Klaraid apenas os termos lineares foram significativos, já para o Novfloc, apenas o termo quadrático da concentração do floculante que não teve significância a $95 \%$. Para se verificar a qualidade de ajuste do modelo, realizou-se a análise dos efeitos e posterior análise de variância (ANOVA), analisando o coeficiente de determinação e o teste $\mathrm{F}$ (Tabela 5).

Avaliando a regressão para ambos os coagulantes, o valor de $F_{\text {calculado }}=13,80$ do Klaraid e $F_{\text {calculado }}=92,32$ do Novfloc foram significativos, com $\mathrm{p}<0,05$ e maior que o $\mathrm{F}_{\text {tab }}(5 ; 5 ; 0,05)=$ 5,02 , ou seja, aproximadamente 2,75 e 18,92 vezes maior respectivamente.

Analisando a falta de ajuste em relação a sedimentação, para o coagulante Novfloc, não foi observada significância para o modelo, pois $\mathrm{F}_{\mathrm{cal}} / \mathrm{F}_{\mathrm{tab}}$ para a falta de ajuste foi inferior a 1 [11]. Em relação ao coagulante Klaraid pode-se perceber que a falta de ajuste se apresentou de forma significativa $F_{\text {cal }}>F_{\text {tab }}$, isto é, o modelo apresentado não é adequado para descrever o comportamento da variável resposta obtida, assim pode-se considerar este modelo como não ideal para validação, pois o desejado seria atingir um termo não significativo, ou seja, um $\mathrm{F}_{\text {calculado }}$ menor do que o $\mathrm{F}_{\text {tabelado }}$.

Com base nos gráficos de superfície de resposta, pode-se observar que não foi encontrada uma faixa de aplicação ótima, e sim uma tendência de uma região que com maiores concentrações de coagulante e floculante, irá se obter uma maior eficiência com relação a remoção de turbidez.

Obseva-se na Figura 4, que os melhores valores de remoção de turbidez foram nas concentrações acima de $427 \mathrm{ppm}$ de coagulante e de 9 ppm de floculante. Assim obteve-se 78,93\%. Em relação à Figura 5 os melhores resultados de remoção foram em concentrações de $427 \mathrm{ppm}$ de coagulante e de 7 ppm de floculante, obtendo 78,09\%.

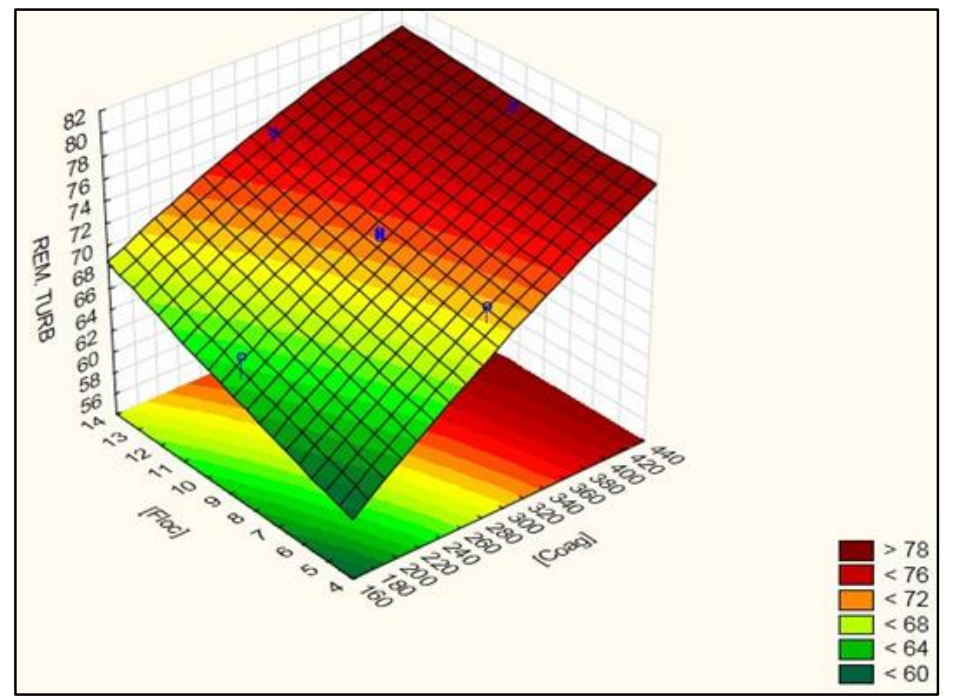

Figura 4 - Gráfico de Superfície Klaraid.

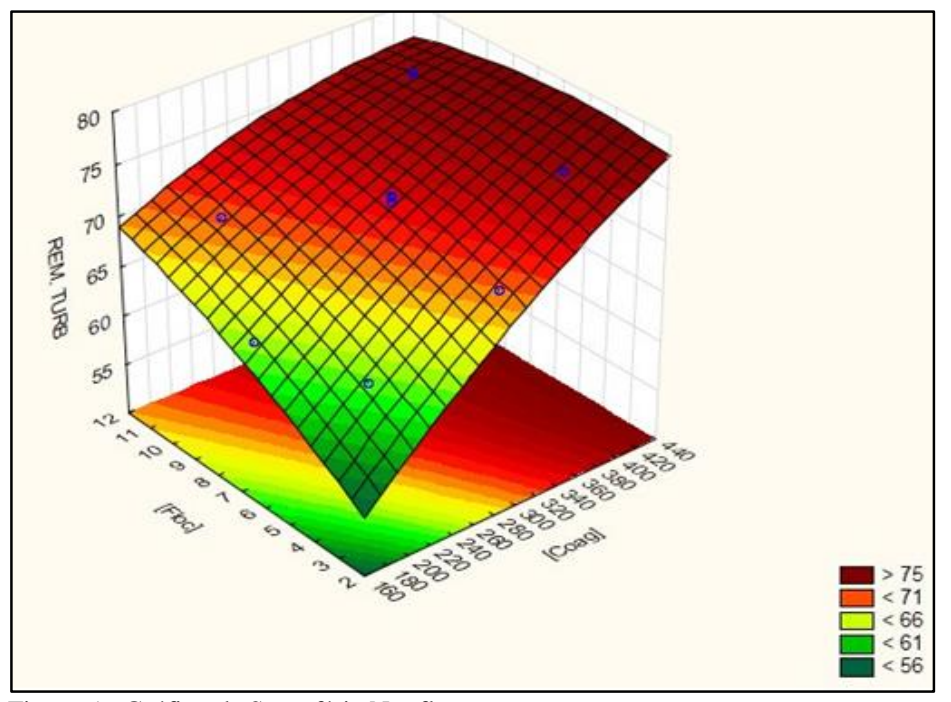

Figura 5 - Gráfico de Superfície Novfloc. 
Tabela 5 - Análise de Variância (95\%).

\begin{tabular}{|c|c|c|c|c|c|c|c|}
\hline & Fonte de Variação & $\begin{array}{c}\text { Soma } \\
\text { Quadrática } \\
\end{array}$ & $\begin{array}{c}\text { Graus de } \\
\text { Liberdade }\end{array}$ & $\begin{array}{c}\text { Média } \\
\text { Quadrática } \\
\end{array}$ & Fcal $(95 \%)$ & $\operatorname{Ftab}(95 \%)$ & Fcal/Ftab \\
\hline \multirow{5}{*}{$\begin{array}{c}\text { Sedimentação } \\
\text { (Klaraid + Polfloc) }\end{array}$} & Regressão (Modelo) & 202,19 & 5 & 40,43 & \multirow{2}{*}{13,80} & \multirow{2}{*}{5,02} & \multirow{2}{*}{2,75} \\
\hline & Resíduos & 14,64 & 5 & 2,92 & & & \\
\hline & Falta de ajuste & 14,51 & 3 & 4,83 & \multirow{2}{*}{73,73} & \multirow{2}{*}{19,2} & \multirow{2}{*}{3,84} \\
\hline & Erro puro & 0,13 & 2 & 0,0 & & & \\
\hline & Total & 216,83 & 10 & & & & \\
\hline \multirow{5}{*}{$\begin{array}{c}\text { Sedimentação } \\
(\text { Novfloc + Polfloc) }\end{array}$} & Regressão (Modelo) & 221,40 & 5 & 44,28 & \multirow{2}{*}{92,32} & \multirow{2}{*}{5,02} & \multirow{2}{*}{18,39} \\
\hline & Resíduos & 2,39 & 5 & 44,28 & & & \\
\hline & Falta de ajuste & 2,19 & 3 & 0,73 & \multirow{2}{*}{7,30} & \multirow{2}{*}{19,2} & \multirow{2}{*}{0,38} \\
\hline & Erro puro & 0,20 & 2 & 0,10 & & & \\
\hline & Total & 223,79 & 10 & & & & \\
\hline
\end{tabular}

Coral, Bergamasco e Bassetti [12] utilizaram o Tanfloc que é de origem vegetal para efluente de galvanoplastia, chegando a bons resultados para concentrações de 400 ppm alcançando remoções de turbidez superiores a 99\%, em ensaios de Jart Test com 1,2 litros de efluente, para tempos e gradientes de $120 \mathrm{rpm}$ para 1,5 minutos, $20 \mathrm{rpm}$ para 15 minutos e 40 minutos de sedimentação.

Santos et al [2] utilizaram os coagulantes inorgânicos, cloreto férrico e sulfato de alumínio, para o tratamento de efluente lácteo, obtendo como resultado eficiências de remoções para turbidez de $97,6 \%$ para o cloreto e $98 \%$ para o sulfato de alumínio para 1 hora de sedimentação.

\subsection{Delineamento Composto Central Rotacional de Flotação}

Visando a otimização do processo da flotação, realizouse um delineamento composto, a faixa de estudo de ambos os coagulantes e floculantes também foi deslocada, assim os níveis foram ampliados e abrangeu-se uma faixa maior.

Como se pode visualizar na Tabela 6 , a turbidez chegou a remoções de $72,11 \%$ e $74,92 \%$ com o Novfloc e Klaraid respectivamente. Com base nestes resultados ajustaram-se os dados para obtenção de um modelo quadrático, relacionando a eficiência de remoção de turbidez. Os modelos ilustrados nas Eq.3 e Eq.4 apresentaram $\mathrm{R}^{2}=0,99164$ e $\mathrm{R}^{2}=0,99033$, significando que $99,16 \%$ e $99,03 \%$ da variação na eficiência de remoção de turbidez é explicada pelo modelo.

Rem. Turbidez pelo Klaraid $=66,55+4,98 \mathrm{Coag}+1,21 \mathrm{Floc}+0,48 \mathrm{Coag}^{2}+$ 0,574 $\mathrm{Floc}^{2}$ (Eq. 3)

Rem. Turbidez pelo Novfloc $=67,33+3,18$ Coag $+1,35$ Floc $+0,312$ Coag $x$ Floc + 0,3158 Floc $^{2}$ (Eq. 4)

Tabela 6 - Matriz do DCCR para Flotação (Níveis Reais e Codificados).

\begin{tabular}{|c|c|c|c|c|c|c|c|}
\hline & Ensaios & Novfloc (ppm) & Polfloc (ppm) & Rem. Turb (\%) & Klaraid (ppm) & Polfloc (ppm) & Rem. Turb (\%) \\
\hline \multirow{4}{*}{ Pontos Fatoriais } & 1 & $210(-1)$ & $7(-1)$ & 63,01 & $210(-1)$ & $7(-1)$ & 61,55 \\
\hline & 2 & $390(1)$ & $7(-1)$ & 68,31 & $390(1)$ & $7(-1)$ & 71,12 \\
\hline & 3 & $210(-1)$ & $13(1)$ & 65,56 & $210(-1)$ & $13(1)$ & 63,01 \\
\hline & 4 & $390(1)$ & $13(1)$ & 72,11 & $390(1)$ & $13(1)$ & 73,31 \\
\hline \multirow{3}{*}{$\begin{array}{l}\text { Repetição no } \\
\text { Ponto Central }\end{array}$} & 5 & $300(0)$ & $10(0)$ & 67,01 & $300(0)$ & $10(0)$ & 66,39 \\
\hline & 6 & $300(0)$ & $10(0)$ & 67,12 & $300(0)$ & $10(0)$ & 66,6 \\
\hline & 7 & $300(0)$ & $10(0)$ & 66,86 & $300(0)$ & $10(0)$ & 66,7 \\
\hline \multirow{4}{*}{ Pontos Axiais } & 8 & $173(-1,41)$ & $10(0)$ & 62,38 & $173(-1,41)$ & $10(0)$ & 60,82 \\
\hline & 9 & $427(1,41)$ & $10(0)$ & 71,96 & $427(1,41)$ & $10(0)$ & 74,92 \\
\hline & 10 & $300(0)$ & $5,8(-1,41)$ & 66,18 & $300(0)$ & $5,8(-1,41)$ & 65,92 \\
\hline & 11 & $300(0)$ & $14,3(1,41)$ & 69,35 & $300(0)$ & $14,3(1,41)$ & 70,24 \\
\hline
\end{tabular}

De acordo com a Figura 6, pode-se visualizar o gráfico da probabilidade normal dos resíduos para ambos os casos, apresentando ajuste satisfatório, pois nos dois casos todos os resíduos estão distribuídos ao redor da reta. Já a Figura 7, apresenta o gráfico dos resíduos pelos valores observados, onde, pode-se visualizar que os valores se apresentam de forma 
aleatória, sem qualquer tendência. As estimativas dos efeitos relacionados à eficiência de remoção de turbidez são ilustradas nos diagramas de Pareto (Figura 8). Os efeitos além da linha tracejada são considerados significativos em um intervalo de 95\% de confiança.

Com base na Figura 8-A e Figura 8-B, pode-se visualizar que para o coagulante Klaraid, tanto os termos lineares quanto os termos quadráticos foram significativos, já para o Novfloc, apenas o termo quadrático da concentração do coagulante que não teve significância a 95\%. Para se verificar a qualidade do ajuste do modelo utilizou-se a análise de variância (ANOVA), analisando o coeficiente de determinação e o teste F (Tabela 7).

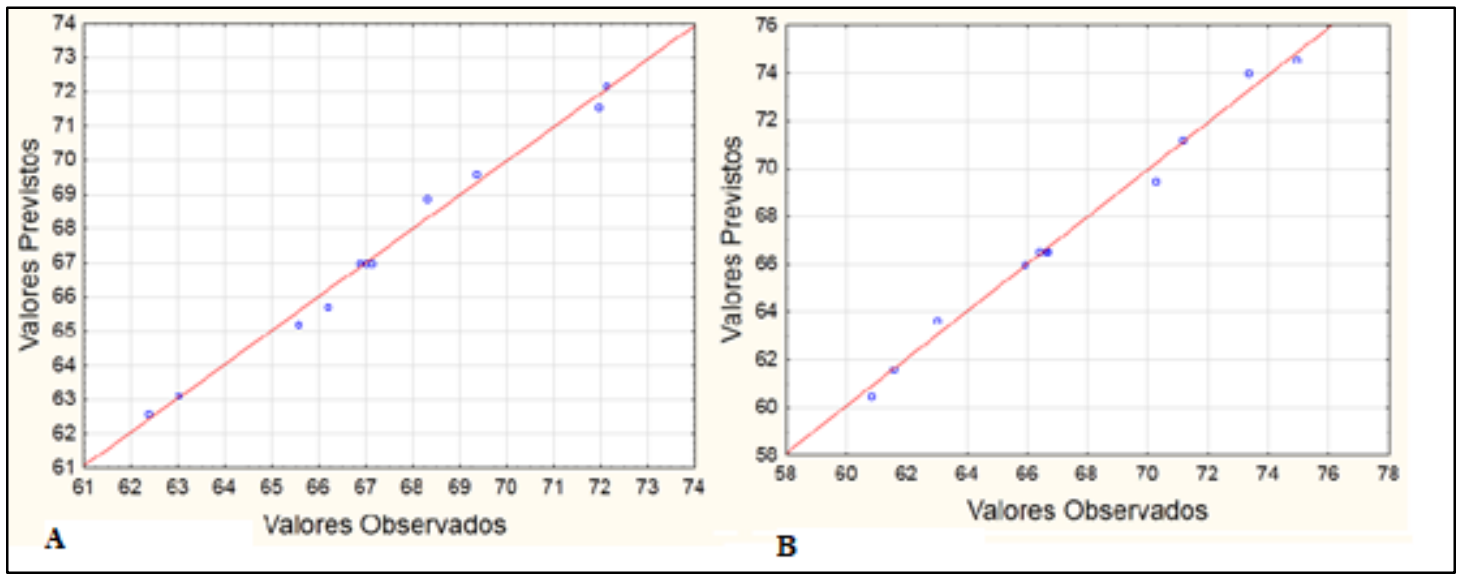

Figura 6 - Gráfico de Probabilidade Normal dos Resíduos (A - Novfloc B - Klaraid).

Avaliando a regressão para ambos os coagulantes, o valor de $F_{\text {calculado }}=118,66$ do Klaraid e $F_{\text {calculado }}=102,37$ do Novfloc foram significativos, com $\mathrm{p}<0,05$ e maior que o $\mathrm{F}_{(5 ; 5 ; 0,05)}=5,02$, ou seja, aproximadamente 23,74 e 20,40 vezes maior respectivamente.

Analisando a falta de ajuste para a flotação, para o coagulante Novfloc não foi observada significância para o modelo, pois $\mathrm{F}_{\mathrm{cal}} / \mathrm{F}_{\text {tab }}$ para a falta de ajuste também foi inferior a 1 [11].

Em relação ao coagulante Klaraid, a falta de ajuste também se apresentou de forma significativa $\left(\mathrm{F}_{\text {calculado }}(5 ; 5 ; 0,05)=22,99>\right.$
$\left.F_{\text {tabelado }}(5 ; 5 ; 0,05)=19,2\right)$, embora o idealizado fosse um valor de

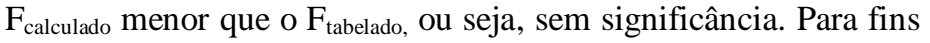
preditivos, tendo como base que a relação do $\mathrm{F}_{\text {cal }} / \mathrm{F}_{\text {tab }}$ foi baixa, pode-se avaliar neste caso a relação entre os pontos centrais e o erro puro gerado na análise de variância. Assim considerando que para remoção de turbidez os pontos centrais foram próximos $(66,38 \% ; 66,59 \%$ e $66,70 \%)$ demonstrando uma boa repetibilidade de resultados, e que o erro puro de 0,05 foi baixo, o modelo pode ser considerado como válido para este caso [11].

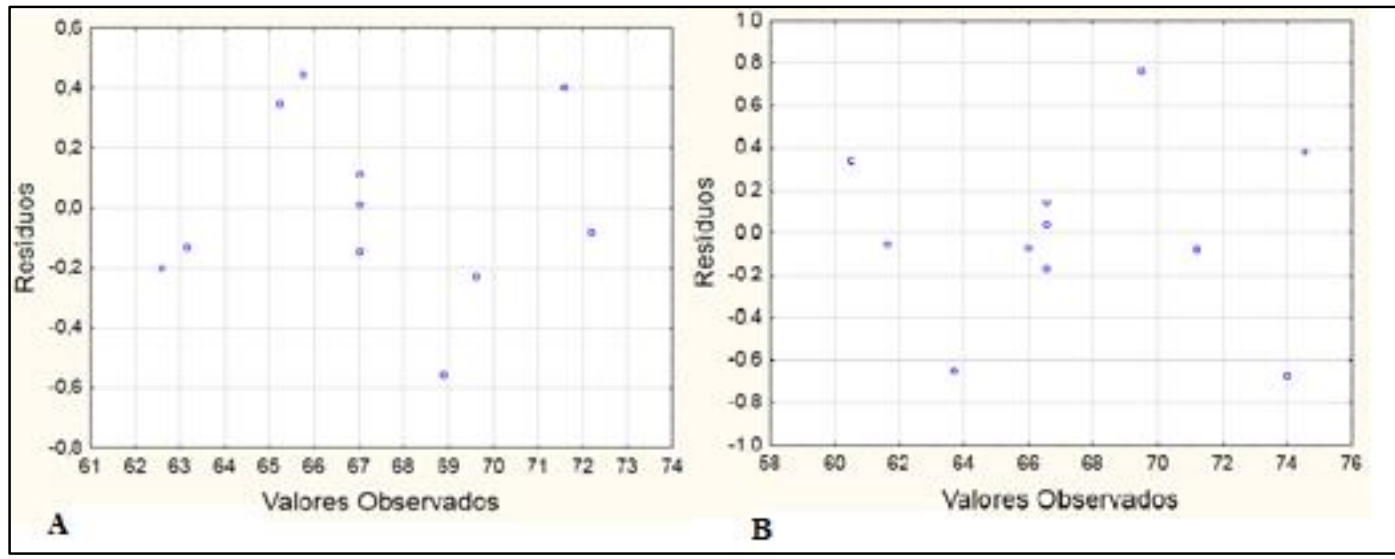

Figura 7 - Gráfico dos Resíduos pelos Valores Observados (A - Novfloc) (B - Klaraid). 

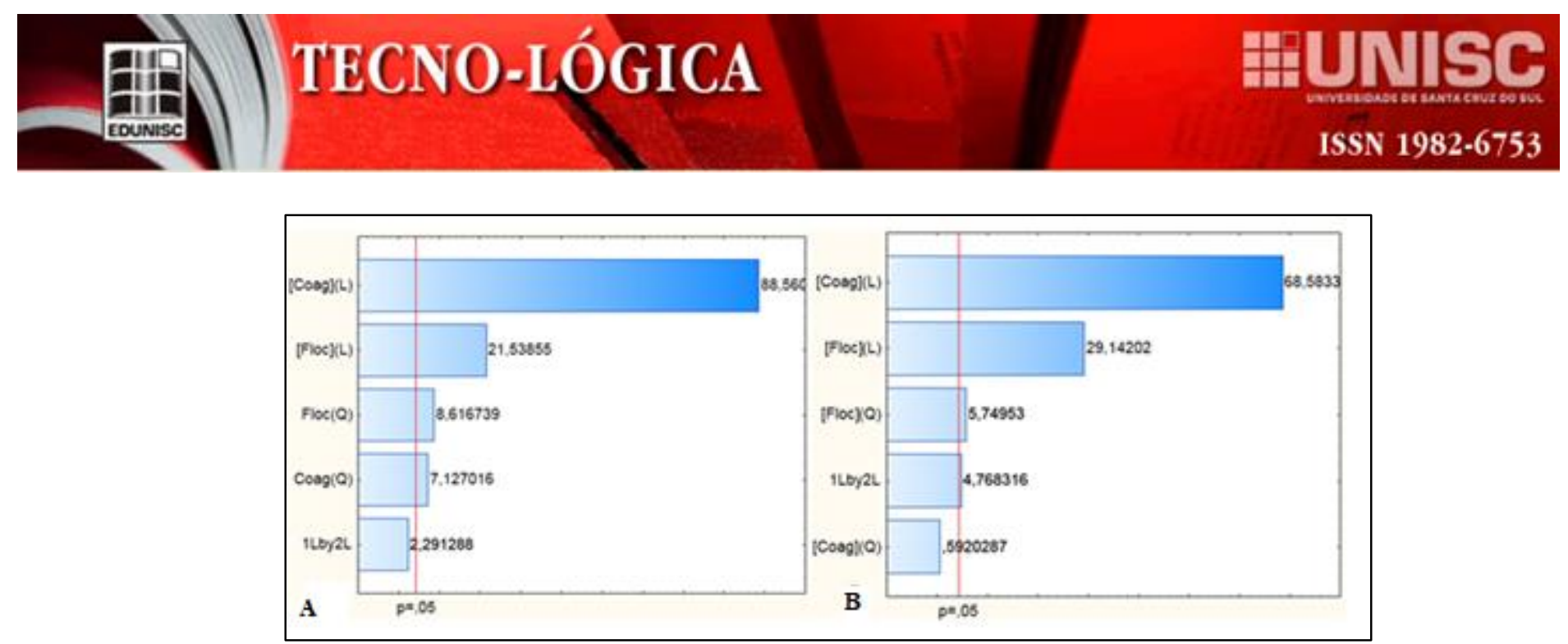

Figura 8 - Diagrama de Pareto (A - Para o Klaraid) (B - Para o Novfloc).

Tabela 7 - Análise de Variância (95\%).

\begin{tabular}{|c|c|c|c|c|c|c|c|}
\hline & Fonte de Variação & $\begin{array}{c}\text { Soma } \\
\text { Quadrática }\end{array}$ & $\begin{array}{l}\text { Graus de } \\
\text { Liberdade }\end{array}$ & $\begin{array}{c}\text { Média } \\
\text { Quadrática }\end{array}$ & Fcal $(95 \%)$ & Ftab $(95 \%)$ & Fcal/Ftab \\
\hline \multirow{5}{*}{$\begin{array}{c}\text { Flotação } \\
(\text { Klaraid + Polfloc) }\end{array}$} & Regressão (Modelo) & 212,69 & 5 & 42,53 & \multirow{2}{*}{118,66} & \multirow{2}{*}{5,02} & \multirow{2}{*}{23,63} \\
\hline & Resíduos & 1,79 & 5 & 0,35 & & & \\
\hline & Falta de ajuste & 1,74 & 3 & 0,58 & \multirow{2}{*}{22,99} & \multirow{2}{*}{19,2} & \multirow{2}{*}{1,19} \\
\hline & Erro puro & 0,05 & 2 & 0,02 & & & \\
\hline & Total & 214,48 & 10 & & & & \\
\hline \multirow{5}{*}{$\begin{array}{c}\text { Flotação } \\
(\text { Novfloc + Polfloc) }\end{array}$} & Regressão (Modelo) & 96,33 & 5 & 19,26 & \multirow{2}{*}{102,37} & \multirow{2}{*}{5,02} & \multirow{2}{*}{20,39} \\
\hline & Resíduos & 0,94 & 5 & 0,18 & & & \\
\hline & Falta de ajuste & 0,90 & 3 & 0,30 & \multirow{2}{*}{17,62} & \multirow{2}{*}{19,2} & \multirow{2}{*}{0,91} \\
\hline & Erro puro & 0,03 & 2 & 0,01 & & & \\
\hline & Total & 97,28 & 10 & & & & \\
\hline
\end{tabular}

Na mesma linha de pensamento Souza e Menezes [13], reforçam que é possível analisar nos casos em que o $\mathrm{F}_{\mathrm{cal}} / \mathrm{F}_{\text {tab }}$ foi baixo, o quadrado médio, assim se o quadrado médio do erro do experimento for baixo, pode ser feita a consideração de que a falta de ajuste é irrelevante para a validação do modelo.

Com base nos gráficos de superfície de resposta, pode-se observar que não foi encontrada uma faixa de aplicação ótima e sim uma tendência de uma região que, com maiores concentrações de coagulantes e floculantes irá se obter uma maior eficiência com relação a remoção de turbidez.

Observa-se na Figura 9, que os melhores valores de remoção de turbidez foram nas concentrações acima de 427 ppm de coagulante e $10 \mathrm{ppm}$ de floculante, assim foi obtido $74,92 \%$. Em relação à Figura 10 os melhores resultados de remoção foram em concentrações de $390 \mathrm{ppm}$ de coagulante e $13 \mathrm{ppm}$ de floculante, obtendo $72,11 \%$.

Casagrande [14] utilizou o PAC como coagulante na flotação para efluente de reatores anaeróbios e obteve resultados positivos na aplicação de $9 \mathrm{mg} . \mathrm{L}^{-1}$ de $\mathrm{Al}_{2} \mathrm{O}_{3}$ de $\mathrm{PAC}$, chegando a remoções $75 \%$ e $70 \%$ de cor e turbidez respectivamente, e com a aplicação de 90 mg. $\mathrm{L}^{-1}$ de $\mathrm{Al}_{2} \mathrm{O}_{3}$ de $\mathrm{PAC}$, obteve grandes eficiências de remoções de $90 \%$ e $97 \%$ de cor e turbidez.

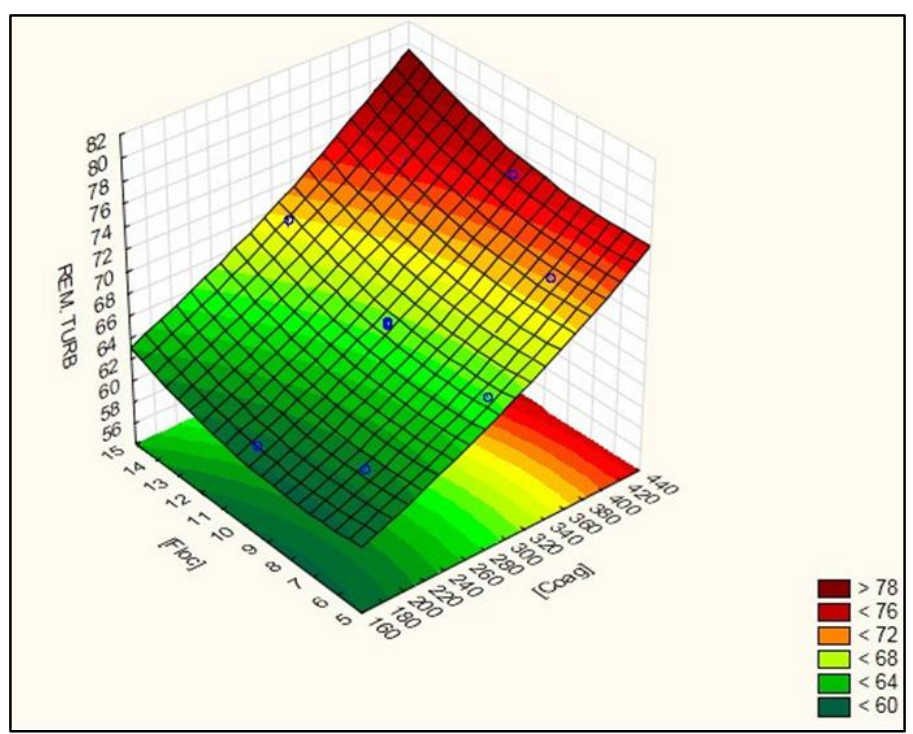

Figura 9 - Gráfico de Superfície Klaraid. 


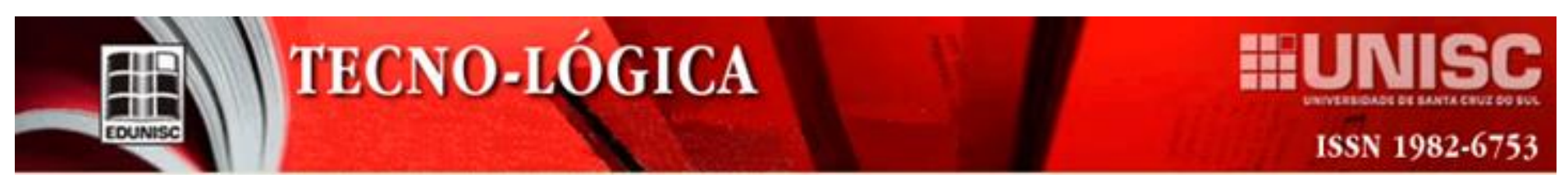

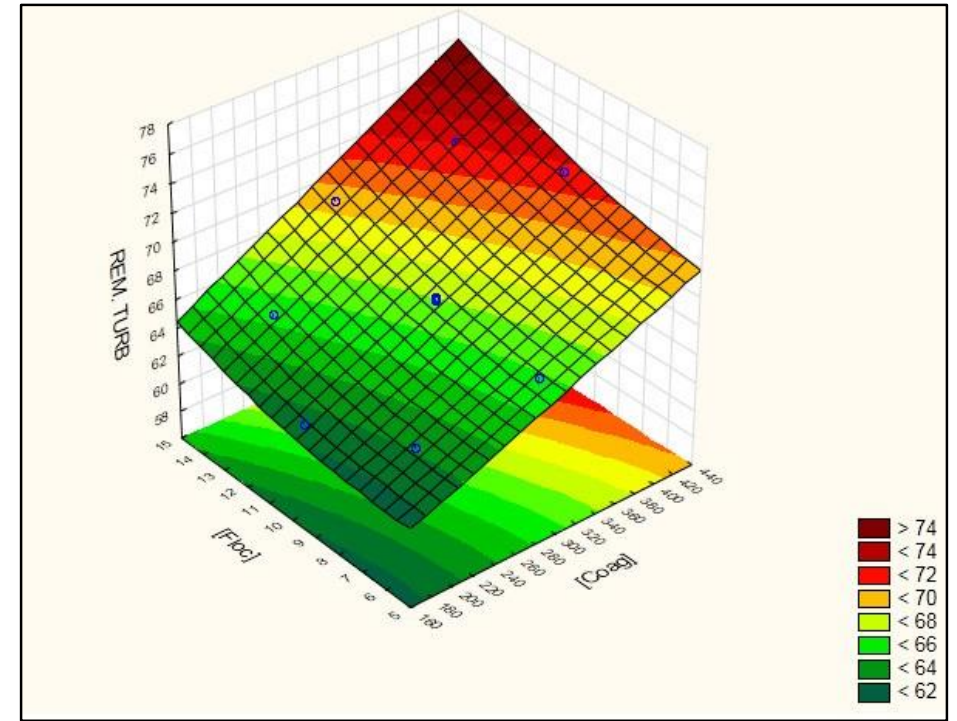

Figura 10 - Gráfico de Superfície Novfloc.

Andrade [15] também utilizou o PAC para os processos de flotação e filtração em efluente de laticínios, com a aplicação de $1400 \mathrm{mg}$. $\mathrm{L}^{-1}$ de $\mathrm{Al}_{2} \mathrm{O}_{3}$ chegou a 90,85\% de remoção de turbidez com a filtração, já para o processo de flotação atingiu remoções de $50,17 \%$ de turbidez.

Os estudos apresentados acima reforçam sobre a eficiência do processo da flotação aplicada tanto em tratamentos de água como de efluentes, utilizando tanto coagulantes inorgânicos como coagulantes com base orgânica. Tendo em vista as avaliações propostas, sabendo que flotação e sedimentação são dois processos que se diferem, pode-se fazer a consideração que neste estudo, houve uma maior eficiência de remoção do parâmetro turbidez, na sedimentação, o que se deu pelas características do efluente e do processo aplicado.

\section{Conclusões}

Com base nos resultados obtidos neste estudo e considerando a condição dos ensaios, pode-se concluir que os coagulantes Novfloc e Klaraid apresentaram resultados similares tanto no processo da sedimentação como no processo da flotação, ambos mostrando ser eficientes na formação de flocos com características apropriadas tanto para o processo de sedimentação como para o processo de flotação.

Tendo em vista que os resultados foram similares pode-se considerar o coagulante com base orgânica como uma alternativa da aplicação dos coagulantes inorgânicos, podendo também auxiliar na geração de um lodo com características biodegradáveis e com um potencial poluidor menor, se comparado ao lodo gerado pela aplicação dos coagulantes inorgânicos.

Para o Novfloc e o Polfloc, os melhores resultados apresentaram uma remoção de turbidez na sedimentação de até
$78,09 \%$, com 390 a 427 ppm de coagulante e de 7 a 10 ppm de floculante, na flotação os resultados foram de $72,11 \%$, com 390 a $427 \mathrm{ppm}$ de coagulante e de 10 a $13 \mathrm{ppm}$ de floculante. Já para a combinação do Klaraid e do Polfloc na sedimentação, os melhores resultados obtiveram remoções de até 78,93\%, com 390 a 427 ppm de coagulante e 9 a $12 \mathrm{ppm}$ de floculante, para flotação os melhores resultados obtiveram até $74,92 \%$, com 390 a 427 ppm de coagulante e 10 a 13 ppm de floculante.

Com relação aos delineamentos compostos de sedimentação e flotação, não foi possível alcançar a faixa ótima da aplicação dos coagulantes na faixa de estudo, apenas uma tendência à melhor dosagem de aplicação.

Como recomendação para trabalhos futuros, com base nos resultados estatísticos, pode-se aumentar as faixas de aplicação dos dois coagulantes avaliados neste estudo, visando à otimização do processo. Com relação aos números de ensaios, recomenda-se aumentar o número de pontos centrais visando uma melhor qualidade na determinação do erro puro. Sugere-se ainda, ampliar o número de variáveis dependentes nos planejamentos como exemplo: cor, DQO, DBO e sólidos totais, para uma determinação mais precisa dos resultados do efluente clarificado.

Por fim recomenda-se estudar e ampliar a pressão aplicada no processo de flotação, pois foi constatado neste estudo que com pressões a baixo de $400 \mathrm{kPa}$, a quantidade de ar saturado apresenta-se insuficiente para a ocorrência do processo da flotação, resultando em flocos que tendem a sedimentar.

\section{POST-PHYSICAL-CHEMICAL TREATMENT BY FAD FLOTATION IN DAIRY EFFLUENTS}

ABSTRACT: The dairy agroindustry is an industry with a high polluting potential effluent that, without proper treatment, becomes an environmental problem. In order to solve the effects of the inadequate treatment of these effluents, complementary to the conventional treatment, in some cases the application of a posttreatment aiming at a better quality of the final effluent is carried out. One of the forms used is flotation preceded by coagulation / flocculation, which through coagulating and flocculating agents allows the agglomeration of particles allowing flotation. These substances are usually chemicals based on aluminum or iron, resulting in the generation of sludge with high concentrations of metallic ions, making it difficult and expensive to use the sludge. Considering the problems mentioned, coagulants and organicbased flocculants have been studied and used since they generate biodegradable sludge. The objective of this work was to compare the efficiency of two coagulants, one inorganic (Klaraid IC 1176L) and the other organic (Novfloc 1540), associated to the flotation and sedimentation process on bench scale, in the treatment of effluent from an agroindustry of dairy. For the accomplishment of this work, the methodology of experimental planning and optimization of processes was applied, using as independent 
variables the concentration of coagulants and flocculants, having turbidity removal as response variable. As a first step, initial factorial $2^{2}$ planning of sedimentation and flotation was done aiming to adjust the application ranges, following a central rotational compound design aiming at the optimization of sedimentation and flotation processes. With the use of the inorganic base coagulant (Klaraid) followed by the flocculant, a turbidity removal of up to 78,93\% was obtained for the sedimentation process, and for the flotation process the best results indicated a turbidity removal of $74,92 \%$. With the use of the organic-based coagulant (Novfloc) combined with the use of the flocculant, the turbidity removal efficiency for sedimentation reached up to $78,09 \%$, while in the flotation the result was up to $72,11 \%$ of removal.

Keywords: Coagulation. Flocculation. Flotation. Effluent. Dairy products.

\section{Referências}

[1] VILLA, R. D.; SILVA, M. R. A.; NOGUEIRA, R. F. P. Potencial de aplicação do processo foto-fenton/solar como pré-tratamento de efluente da indústria de laticínios, Quim. Nova. Vol. 30, n. 8, p.1799-1803, 2007.

[2] SANTOS, A. M. et al. Tratamento de efluentes lácteos através de coagulação química e sedimentação. VIII Congresso Brasileiro de Engenharia Química em Iniciação Científica. Uberlândia, MG. 2009.

[3] KUSHWAHA, J. P.; SRIVASTAVA, V. C.; MALL, I.D. Treatment of dairy wastewater by commercial activated carbon and bagasse fly ash: Parametric, Kinetic and equilibrium modeling, disposal studies, Bioresource Technology. Vol. 1001, n. 10, p. 3474-3483, 2010.

[4] DA SILVA, T. E. M. et al. Avaliação do uso de coagulantes orgânicos no tratamento de efluentes de galvanoplastia: UM ESTUDO DE CASO. Blucher Chemistry Proceedings, Vol. 3, n. 1, p. 1122-1128, 2015.

[5] NUNES, J. A. Tratamento físico-químico de águas residuárias industriais. 6. ed. Aracaju: J. Andrade, 2012.

[6] SCHMITT, D. M. F. Tratamento de águas residuárias da indústria de Laticínios pelos processos combinados coagulação/floculação/ adsorção/ ultrafiltração utilizando semente de moringa oleífera como coagulante. Dissertação (Mestrado em Engenharia Química, área de concentração em Desenvolvimento de Processos) - Universidade Estadual do Oeste do Paraná. Toledo - PR. 2011.

[7] DI BERNARDO, L.; DI BERNARDO, A.; CENTURIONE FILHO, P. L. Ensaios de Tratabilidade de Água e dos Resíduos Gerados em Estações de Tratamento de Água. São Paulo: Rima, 2002.

[8] SOLANA, I. Estudo da viabilidade de utilização de um polímero de base orgânica em substituição ao cloreto férrico no tratamento de efluente industrial. Trabalho de Conclusão de Curso - Universidade Tecnológica Federal do Paraná. Medianeira. 2014.

[9] CENTURIONE FILHO, P. L.; DI BERNARDO, L. Procedimento para execução de ensaios de flotação/filtração em equipamento de bancada. Revista de Engenharia Sanitária e Ambiental. Vol. 8, n. 1, p. 39-44, 2003.
[10] FONTANELlA, D. F. Performace de ETE: otimização da estação de tratamento de efluentes da indústria cerâmica. Trabalho de Conclusão de Curso Universidade do Extremo Sul Catarinense. Criciúma. 2003.

[11] BARROS NETO, B.; SCARMINIO, I. S.; BRUNS, R. E. Como fazer experimentos: pesquisa e desenvolvimento na ciência e na indústria, $3^{\mathrm{a}}$ ed., Ed. Da Unicamp: Campinas, São Paulo, 2007.

[12] CORAL, L. A.; BERGAMASCO, R.; BASSETTI, F. J. Estudo da viabilida de de utilização do polímero natural (TANFLOC) em substituição ao sulfato de alumínio no tratamento de águas para consumo. In: INTERNATIONAL WORKSHOP ADVANCES IN CLEANER PRODUCTION. 2009. p. 20-22.

[13] SOUZA, M. L.; MENEZES, H. C. Otimização do processo de extrusão termoplástica da mistura castanha do Brasil com farinha de mandioca. Revista Ciência Tecnologia de Alimentos, v.28, n.3, p.659-667, jul.-set. 2008.

[14] CASAGRANDE, L. E. M. Sistema de bancada de flotação por ar dissolvido e filtração-adsortiva para tratamento de efluentes de reatores anaeróbios. Monografia (Trabalho de Conclusão do Curso Superior em Engenharia Ambiental). Universidade Tecnológica Federal do Paraná. Londrina, PR, 2014, 63p.

[15] ANDRADE, L. H. Tratamento de efluente de indústria de laticínios por duas configurações de biorreator com membranas e nano filtração visando o reuso. Dissertação (Mestrado em Saneamento, Meio ambiente e Recursos Hídricos) Universidade Federal de Minas Gerais. Belo Horizonte - MG. 2011., Brazil. 